fibrosis using Fib-4 did not attend specialist follow up and represent a missed opportunity for intervention. The basis of our Quality Improvement Project.

\section{PWE-49 ROUTINE PRE-PROCEDURE BLOOD TESTS ARE NOT REQUIRED TO RUN A SAFE NURSE LED PARACENTESIS SERVICE}

Alison Wickham*, Ben Johnson, Andrew King. University Hospitals Birmingham, Birmingham, UK

\subsection{6/gutjnl-2021-BSG.239}

Introduction Patients with liver cirrhosis and ascites who require large volume paracentesis (LVP) may have an abnormal coagulation profile, with prolongation of the prothrombin time and thrombocytopenia. Current guidelines do not advocate the use of clotting factors or platelets prior to LVP. In 2017 we established a nurse led day case paracentesis service, pre procedure bloods were not routinely checked and drains inserted without imaging guidance.

Methods A retrospective review of patients undergoing LVP within our service between 2017-2019 was undertaken. All procedures were undertaken independently by 2 trained nurse practitioners. The results of blood tests taken, to the nearest date of each procedure, were used for analysis but not for routine practice. Any procedural complications were documented.

Results Fifty seven patients were identified who required a total of 239 LVP's over the time period. $91.6 \%$ of the procedures were successfully completed. $73 \%$ had alcohol related liver disease and median UKELD score was 54 (range 45-70).

$69 \%$ of patients had their bloods checked more than 1 week prior to LVP and $21 \%$ of those were more than 4 weeks prior to procedure.

1 patient received 1 unit platelet transfusion, platelet count was 64 and no indication for transfusion was identified from the records.

No bleeding related complications occurred. 2 minor complications were recorded - one drain site cellulitis, one persistent drain site leak.

Conclusions Non medical paracentesis without routine pre procedure bloods is safe and effective, even in the presence of coagulopathy of liver disease and renal failure. This data sup-

\begin{tabular}{lcc} 
Abstract PWE-49 Table 1 & blood parameters prior to LVP $(\mathrm{n}=239)$ \\
\hline & Median & Range \\
\hline INR & 1.3 & $1.0-$ \\
& & 3.2 \\
Platelets $\left(\times 10^{9} /\right)$ & 137 & $34-$ \\
& & 331 \\
Creatinine (umol/ & 78 & $41-$ \\
I) & & 814 \\
\hline
\end{tabular}

ports the implementation of published guidelines into nurse led services.

\section{PWE-50 DEPENDENCE OF BMI ON C677T AND A1298C POLYMORPHISMS OF METHYL ENTRAHYDRAFOLATREDUCTASE-GENE IN PATIENTS WITH NON-ALCOHOLIC-FATTY-LIVER-DISEASE} 1,2Toufik Abdul-Rahman*, ${ }^{1,2}$ Andrew Awuah Wireko, ${ }^{1}$ Nataliia Kuchma, 'Shekinah
Amaka Obinna. 'Sumy State University, Ukraine; ${ }^{2}$ Toufik's World Organization, Ukraine

10.1136/gutjnl-2021-BSG.240

Introduction Non-alcoholic fatty liver disease (NAFLD) occurs in almost $50 \%$ of the adult population and is the cause of liver dysfunction in developed countries. The widespread use of overweight makes the problem of NAFLD relevant among both adults and children. The vast majority of patients with NAFLD are overweight and obese, which increases the risk of developing atherosclerosis, hypertension and type 2 diabetes and accelerates the progression of pathological changes in the liver. Genes associated with the onset and progression of liver disease and obesity are widely studied today. There is evidence that the methylenetetrahydrofolate reductase (MTHFR) gene affects the development and progression of NAFLD and hyperhomocysteinemia, associated with allelic polymorphism of this gene affects the development of pathological changes in the liver and in the body as a whole.

Aim The aim of our study was to investigate the dependence of BMI on C677T and A1298C polymorphisms of the MTGFR gene in patients with NAFLD.

Method We monitored 110 patients with NAFLD. The diagnosis of NAFLD was made on the basis of laboratory and instrumental methods of examination. The calculation of BMI was performed according to the formula Kettle - weight $(\mathrm{kg}) /$ height $(\mathrm{m})^{2}$. Determination of allelic polymorphism was performed by PCR with detection of results by hybridization-fluorescence method in real time.

Results As a result of our studies, we found that carriers of genotypes C677C, C677T and T677T of the MTGFR gene had BMI values of $34.7 \pm 3.6,37.9 \pm 3.8$ and $39.7 \pm 3.2$ $\mathrm{kg} / \mathrm{m} 2$. We found that patients carrying the T-allele $(\mathrm{C} / \mathrm{T}$ and $\mathrm{T} / \mathrm{T}$ genotypes) had significantly higher BMIs compared to homozygotes for the main $\mathrm{C}$-allele (C/C genotype). Studying the frequency of genotypes by $\mathrm{A} 1298 \mathrm{C}$ polymorphism of the MTGFR gene depending on BMI, it was found that carriers of genotypes A1298A, A1298C and C1298C had BMI values of $37.1 \pm 4.1,36.6 \pm 4.2$ and $36.9 \pm 3.8 \mathrm{~kg} / \mathrm{m} 2$ in accordance. We did not find a significant difference in the frequency distribution of genotypes by A1298C polymorphism depending on BMI

Conclusions BMIs are favorably associated with the frequency of genotypes by the C677T polymorphism of the MTGFR gene and do not have such an association with the frequency of genotypes by the A1298C polymorphism of the MTGFR gene. 